\title{
SPATIO-TEMPORAL CHANGE MODELING OF LULC: A SEMANTIC KRIGING APPROACH
}

\author{
Shrutilipi Bhattacharjee ${ }^{\mathrm{a}}$, Soumya K. Ghosh ${ }^{\mathrm{b} *}$ \\ ${ }^{\mathrm{ab}}$ School of Information Technology, Indian Institute of Technology Kharagpur, West Bengal-721302, India \\ Email: a shrutilipi.2007@ gmail.com, ${ }^{b}$ skg@iitkgp.ac.in
}

KEY WORDS: Remote sensing, Prediction, Kriging, LULC, Data semantic, Ontology

\begin{abstract}
:
Spatio-temporal land-use/ land-cover $(L U L C)$ change modeling is important to forecast the future $L U L C$ distribution, which may facilitate natural resource management, urban planning, etc. The spatio-temporal change in $L U L C$ trend often exhibits non-linear behavior, due to various dynamic factors, such as, human intervention (e.g., urbanization), environmental factors, etc. Hence, proper forecasting of $L U L C$ distribution should involve the study and trend modeling of historical data. Existing literatures have reported that the meteorological attributes (e.g., $N D V I, L S T, M S I$ ), are semantically related to the terrain. Being influenced by the terrestrial dynamics, the temporal changes of these attributes depend on the LULC properties. Hence, incorporating meteorological knowledge into the temporal prediction process may help in developing an accurate forecasting model. This work attempts to study the change in inter-annual $L U L C$ pattern and the distribution of different meteorological attributes of a region in Kolkata (a metropolitan city in India) during the years 2000-2010 and forecast the future spread of LULC using semantic kriging (SemK) approach. A new variant of time-series $\operatorname{SemK}$ is proposed, namely $\mathrm{Rev}-\mathrm{Sem} K_{\mathrm{ts}}$ to capture the multivariate semantic associations between different attributes. From empirical analysis, it may be observed that the augmentation of semantic knowledge in spatio-temporal modeling of meteorological attributes facilitate more precise forecasting of $L U L C$ pattern.
\end{abstract}

\section{INTRODUCTION}

One of the major challenges associated in monitoring the environmental changes is to predict the meteorological pattern with highest degree of accuracy. Further, forecasting the land-use/ land-cover $(L U L C)$ change will help in assessing the impact of urbanization, city planning, and other socio-economic activities. It has direct impact on several environmental threats, such as: urban heat island, drought, flood, etc. Several studies have reported that the meteorological attributes (for example, land surface temperature (LST), normalized difference vegetation index (VI), moisture stress index $(M S I)$, etc.), that are near to the earth surface are influenced by the behavior of $L U L C$, and positively correlated with its distribution pattern [(Hengl et al., 2012) (Bhattacharjee et al., 2014)]. Hence, their spatio-temporal analysis may incorporate the knowledge of $L U L C$ by semantic analysis of the terrain to achieve better accuracy. Therefore, the meteorological attributes and the $L U L C$ can be regarded as the inter-dependent factors of the terrain, which together influence the environmental changes. This work focuses on the spatio-temporal forecasting of land-use/ land-cover $(L U L C)$ distribution pattern of a spatial region, by learning the past behaviors of meteorological data. It has been hypothesized by the scientists that the twenty first century will be an era of predicting the weather/ meteorological patterns with some well-defined mathematical equations (Shukla, 1998). However, the model which can deal with all the spatial uncertainties with high accuracy is lacking. Though prediction/ forecasting of meteorological attributes have been studied extensively in the field of remote sensing and geographic information system, however accuracy is still a major research challenge. In most of the cases of meteorological forecasting, the auxiliary information in the terrain are overlooked, which have a huge impact on the primary attribute to be predicted, and often contribute to achieve better accuracy. Hence, the shift of research paradigm from univariate to multivariate scenario is indispensable for the accurate mapping of the environmental changes.

\footnotetext{
${ }^{*}$ Corresponding author
}

In order to carry out a data driven approach for analyzing the past behavioral pattern of the meteorological attributes, the kriging (Humme et al., 2006) based interpolation methods have been reported as the most popular, widely used, and best suitable techniques in literature, for handling spatial variability of the terrain. One such way to deal with this variability is to make use of other secondary information while predicting the actual or the primary variable/ attribute. In that case, the secondary variables have to be correlated with primary and must influence it significantly. In the literatures of geo-statistical kriging estimators, the commonly known approaches which can accommodate these multivariate information into the interpolation process are co-kriging, kriging with external drift, regression kriging, etc. However, most of these methods (both univariate and multivariate) have ignored to model the semantic $L U L C$ knowledge of the terrain for predicting meteorological attributes. In previously proposed interpolation methods, namely semantic kriging (SemK) (Bhattacharjee et al., 2014) and times-series semantic kriging $\left(\right.$ Sem $\left._{\mathrm{ts}}\right)$ (Bhattacharjee and Ghosh, 2015) , the $L U L C$ based knowledge is incorporated in the prediction equation for better (accurate) estimation of different meteorological attributes. The traditional two dimensional spatial auto-correlation model, semivariogram has been extended further in spatio-semantic semivariogram model, for analyzing, quantifying the impact of semantic $L U L C$ knowledge over other meteorological attributes. The proposed work also focuses on the spatio-temporal forecasting, however, the distribution of $L U L C$ has been considered as the primary variable to be predicted. The proposed work is a new interpolation based approach, namely Rev-SemK $K_{\mathrm{ts}}$, which can be regarded as the reverse times-series semantic kriging, where different meteorological attributes are considered as the auxiliary information for predicting the landcover pattern. Hence, the proposed approach belongs to the family of multivariate kriging, where $L U L C$ pattern is the primary prediction attribute, and the two other correlated meteorological attributes (Bhattacharjee and Ghosh, 2015), namely land surface temperature (LST) and normalized difference vegetation index $(N D V I)$ have been considered as the secondary information. The 
broad objectives of the work can be stated as follows:

- extending the times-series semantic kriging in multivariate scenario for the augmentation of the auxiliary spatial information of the terrain

- modeling a reverse $S e m K$ approach, $R e v-S e m K_{\mathrm{ts}}$, for quantifying the temporal change of semantic knowledge (or, the change in $L U L C$ pattern) with respect to other meteorological attributes

- modeling the spatio-temporal semivariograms and cross- semivariograms for analyzing the correlation among different factors

- modeling the estimation equation for Rev-SemK $K_{\mathrm{ts}}$ to forecast the $L U L C$ distribution pattern in future time instances

- experimentation with real meteorological data, to demonstrate the efficacy of the proposed method

The rest of the paper is organized as follows. Section 2. presents the state of the art related to the $L U L C$ change modeling, forecasting of $L U L C$ pattern, utilizing this knowledge for the prediction of meteorological attributes, etc. The preliminary works on semantic kriging, and its time-series extension are presented in the Section 3. The framework on forecasting of the future $L U L C$ pattern using the proposed $\operatorname{Rev}-\operatorname{Sem} K_{\mathrm{ts}}$ approach is presented in Section 4. The experimental results, yield by the proposed approach are presented in Section 5. Finally, the conclusion is drawn in the Section 6

\section{BACKGROUND}

Many literatures have reported the impact of $L U L C$ change modeling for the detection of global environmental dynamism, operational planning of different biospheric, atmospheric aspects, urban and city planning, disaster management, etc. Ellis et al. (Ellis and Porter-Bolland, 2008) have compared the change of $L U L C$ in two neighbouring study areas in the Central Yucatan Peninsula Region of Mexico. They have discussed about the dominant pathways in $L U L C$ and the factors influencing deforestation and forest conservation processes occurring in the study region. Schilling et al. (Schilling et al., 2010) have studied how the change in $L U L C$ influences the rapid expansion of soybean cultivation in the Mississippi River basin, and found it to be highly influential for the regional water and climate patterns. Muñoz-Villers et al. (Muñoz-Villers and López-Blanco, 2008) have monitored the $L U L C$ changes between 1990 and 2003 in a tropical mountainous watershed using Landsat TM imagery. They have used a RS-GIS approach and achieved above $75 \%$ classification accuracy. Hoek et al. (Hoek et al., 2008) have used the land-use regression methods for modeling annual mean concentrations of different meteorological attributes such as: $\mathrm{NO}_{2}, \mathrm{NO}_{x}, \mathrm{PM}_{2.5}$, etc. in European and North-American cities.

A very few works have been reported on modeling the $L U L C$ knowledge for the interpolation based prediction of meteorological attributes, and vice-versa. Though some of the articles state the importance of this knowledge for different meteorological analysis, however they fail to quantify and incorporate this property of the terrain into the prediction process. Hengl et al. (Hengl et al., 2012) have reported the land-cover pattern to be one of the influencing factor for predicting the land surface temperature trend. Several other literatures [(Lambin and Geist, 2006) (Mahmood et al., 2010) (Sertel et al., 2011)] have also mentioned about the significance of this knowledge for the weather/ climatic patterns and their dynamics. Janssen et al. (Janssen et al., 2008) have proposed a detrended kriging model, namely $R I O$, for air pollution measurement. They have used CORINE LULC data for developing the land-use indicator. Petrişor et al. (Petrişor et al., 2010) have examined whether a study region is affected at macro-scale through the changes of land-cover or land-use. They have used ordinary kriging method for capturing the environmental changes and their effect on land-use. As far as our knowledge is concerned, no such article have been reported regarding the reverse analysis of meteorological attributes, such as: $L S T, N D V I$, etc., using multivariate spatial interpolation for forecasting the future $L U L C$ pattern. The previous works on semantic kriging [(Bhattacharjee et al., 2014) (Bhattacharjee and Ghosh, 2014)] are one of the initial attempts to incorporate this knowledge into the interpolation process for meteorological attribute prediction. It has been further extended to temporal domain, resulting univariate times-series semantic kriging $\left(\mathrm{SemK}_{\mathrm{ts}}\right)$ (Bhattacharjee and Ghosh, 2015), for forecasting. The proposed method can be considered as the reverse $S e m K_{\mathrm{ts}}$ process in multivariate scenario. The overall idea of the proposed approach and its relationship with the existing works on semantic kriging are depicted in Fig 1. The subsequent sections present the overview of the basic SemK technique, mainly on the quantification of semantic $L U L C$ knowledge, and its temporal extension.

\section{SEMANTIC KRIGING (SEMK) AND ITS TIME-SERIES EXTENSION}

The semantic kriging $(\mathrm{SemK})$, initially proposed in (Bhattacharjee et al., 2014), attempts to quantify the semantic LULC knowledge of the terrain for the prediction of meteorological attributes through spatial interpolation. It extends the traditional semivariogram model into a three dimensional spatio-semantic semivariogram, by incorporating the distribution pattern and influence of the surrounding spatial feature for the variance modeling. One of the most popular kriging based method ordinary kriging $(O K)$ (Stein, 1999) has been extended further with the quantified semantic knowledge for modeling the relatedness between the sample points. For quantifying this new knowledge of the terrain, the association between the representative spatial features of the sample points is mapped such that it adhere to the Tobler's law of spatial proximity (Tobler, 1970). That is, the semantically similar and the correlated features should be assigned more weightage than a dissimilar feature, and vice-versa. This association between different land-cover classes are measured by organizing them into an ontology hierarchy [(Bhattacharjee et al., 2014) (Bhattacharjee et al., 2012)]. It is built with the exhaustive feature set ( $L U L C$ classes) of the spatial region of interest $(R o I)$. Figure 2 presents an example ontology hierarchy for the spatial study region Kolkata, India. It is constructed with reference to the $L U L C$ classification classes, reported by NRDMS, India in (Mendiratta et al., 2008). The "is-a" (hyponym) semantic relation is considered here to map the type of association between classes, however, any semantic relation could have been used, depending on the application requirement.

For the quantification of this semantic knowledge, each of the sampled locations are then mapped to the most appropriate representative leaf feature in the hierarchy. Two metrics have been proposed for analyzing the association between a pair of leaf features in the hierarchy, namely: semantic similarity and the spatial importance. For the actual interpolation process, these two metrics are used for mapping the traditional covariance measure into higher semantic dimension. 


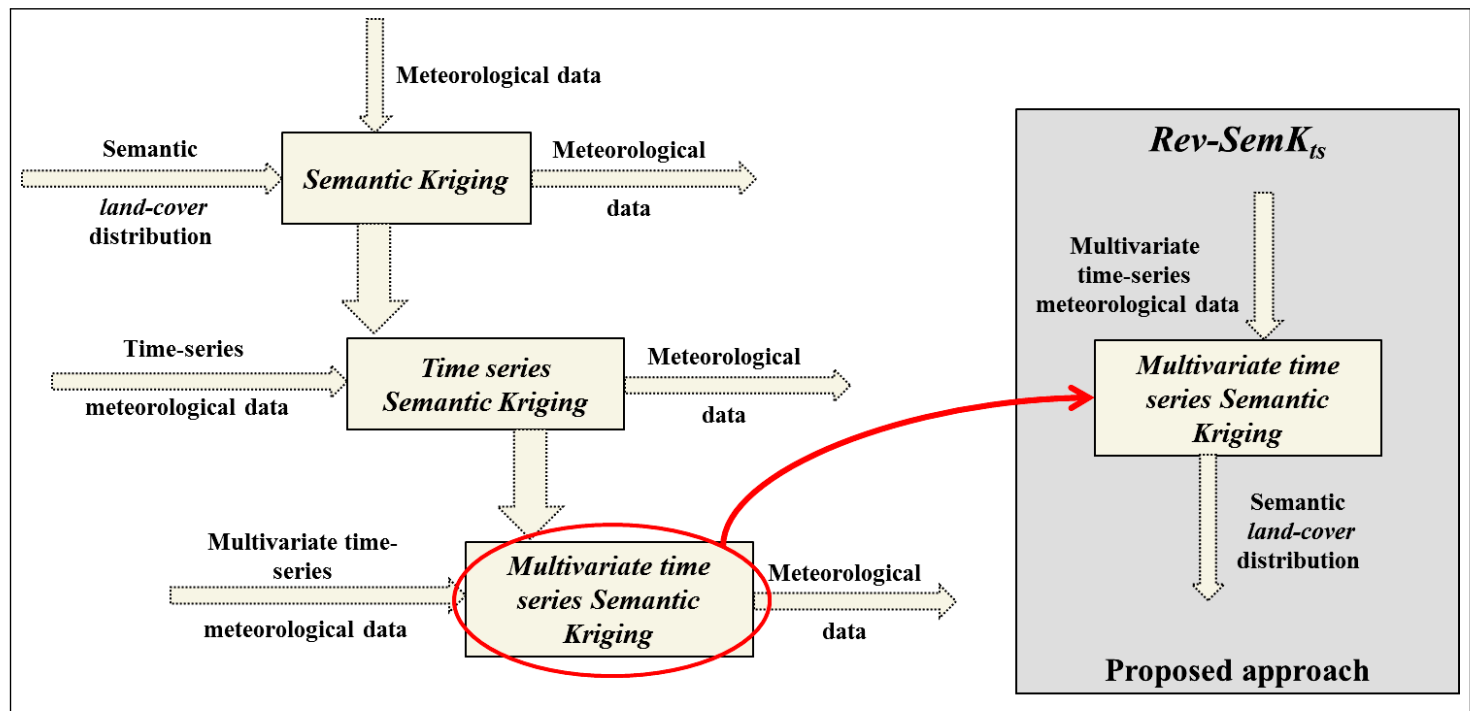

Figure 1. Relation of $\operatorname{Sem} K, \operatorname{Sem} K_{\mathrm{ts}}$ and $\operatorname{Rev}-\operatorname{Sem} K_{\mathrm{ts}}$

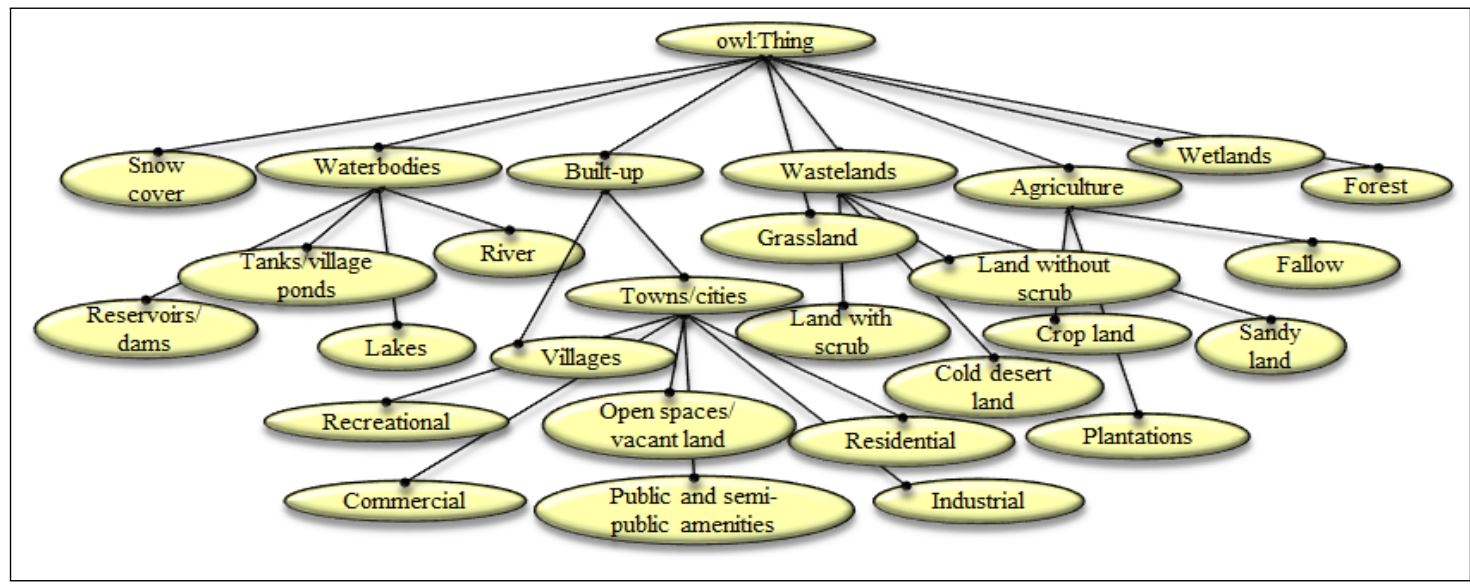

Figure 2. Spatial feature ontology for meteorological attribute prediction (Bhattacharjee and Ghosh, 2015)

\subsection{Semantic Similarity}

This metric is analyzed between any pair of sample points or their representative leaf $L U L C$ classes in the ontology hierarchy. Modified context resemblance method (Manning et al., 2008) have been used here for modeling the metric. The semantic similarity between the $i^{t h}$ and $j^{t h}$ sample points (or their representative features $f_{i}$ and $f_{j}$ referring the ontology) is denoted as $S S_{i j}$, given as follows:

$$
S S_{i j}=\frac{\frac{m_{i}}{\left|f_{i}\right|}+\frac{m_{j}}{\left|f_{j}\right|}}{2}
$$

where, $\left|f_{i}\right|$ and $\left|f_{j}\right|$ are the total number of $L U L C$ classes in the $i^{t h}$ and $j^{t h}$ feature paths; $m_{i}$ and $m_{j}$ are the number of $L U L C$ classes matching in both the paths in the ontology, respectively.

\subsection{Spatial Importance}

This metric is more pragmatic one as it considers the real-time sample points from the terrain. Hence this metric may change with respect to the study region, time, and the attribute to be predicted. The spatial importance between each pair of leaf $L U L C$ classes is measured by the correlation analysis between the sampled locations, represented by these features with respect to the prediction attribute. First, the entire RoI is divided into $k$ number of non-overlapping zones $\left(R_{k}\right)$ such that $\bigcup_{i=1}^{k} R_{k}=R o I$ and $k$ pairs of sample points (representing the pair of features) are chosen from each of the zones. Next, the pairwise correlation score is measured with these $k$ pairs of sample points, which is termed as spatial importance between the pair of features. The spatial importance between $i^{\text {th }}$ and $j^{\text {th }}$ sample points, or their representative features $f_{i}$ and $f_{j}$ in the ontology, is denoted as $S I_{i j}$ and is given as follows:

$$
\begin{aligned}
S I_{i j} & =\operatorname{Corr}_{M P}\left(f_{i}, f_{j}\right) \\
& =\frac{\sum_{m=1}^{k}\left(Z\left(f_{i_{m}}\right)-\overline{Z\left(f_{i}\right)}\right)\left(Z\left(f_{j_{m}}\right)-\overline{Z\left(f_{j}\right)}\right)}{\sqrt{\sum_{m=1}^{k}\left(Z\left(f_{i_{m}}\right)-\overline{Z\left(f_{i}\right)}\right)^{2} \sum_{m=1}^{k}\left(Z\left(f_{j_{m}}\right)-\overline{Z\left(f_{j}\right)}\right)^{2}}}
\end{aligned}
$$

where, $Z\left(f_{p_{q}}\right)$ represents the random field value of the $q^{\text {th }}$ sample point, representing the feature $f_{p} ; \overline{Z\left(f_{p}\right)}$ represents the average of the random field values of the feature $f_{p}$, over $k$ sample points; MP is the meteorological attribute to be predicted.

\subsection{Times-series Semantic Kriging $\left(\operatorname{SemK}_{\mathrm{ts}}\right)$}

For the time-series augmentation of semantic kriging approach, the above mentioned two semantic metrics, namely semantic similarity and spatial importance (Bhattacharjee et al., 2014), are 
extended in temporal dimension. This modeling is presented in (Bhattacharjee and Ghosh, 2015). Between the two semantic metrics, the evaluation of the semantic similarity solely depends on the structure of the ontology hierarchy. As this hierarchy is built with the exhaustive spatial feature set, it remains static with respect to time, hence the time-series covariance (more generally the relatedness) between any $L U L C$ classes with respect to this metric would be same with the spatial covariance between them. Therefore, its temporal evaluation (time-series semantic similarity) will be same with the spatial semantic similarity (Bhattacharjee et al., 2014)

However, as the spatial importance deals with real-time data for its evaluation process, the significance of temporal lag between the sample points is highly evident for this metric. Given any two sampled locations $\left(x_{i}, t_{-i}\right)$ and $\left(x_{j}, t_{-j}\right)$, represented by $f_{i}$ and $f_{j}$ land-cover classes respectively, the whole study region is again divided into $k$ number of random zones. The $k$ pairs of sample points are chosen and measured for both $f_{i}$ and $f_{j}$, but from different time instances, $t_{-i}$ and $t_{-j}$, respectively. The correlation analysis is carried out between these $k$ pairs. The temporal extension of the spatial importance metric is termed as time-series importance $\left(\mathbf{S I}^{t s}\right)$. Hence, the time-series importance between any $i^{\text {th }}$ and $j^{\text {th }}$ sample points (or their representative LULC classes $f_{i}$ and $f_{j}$ ) is evaluated as follows:

$$
\begin{aligned}
& S I_{i j}^{t s}=\operatorname{Corr}_{M P}\left(f_{i}^{t_{-i}}, f_{j}^{t_{-j}}\right)= \\
& \frac{\sum_{m=1}^{k}\left(Z^{t_{-i}}\left(f_{i_{m}}\right)-\overline{Z^{t_{-i}}\left(f_{i}\right)}\right)\left(Z^{t_{-j}}\left(f_{j_{m}}\right)-\overline{Z^{t_{-j}}\left(f_{j}\right)}\right)}{\sqrt{\sum_{m=1}^{k}\left(Z^{t_{-i}}\left(f_{i_{m}}\right)-\overline{Z^{t_{-i}}\left(f_{i}\right)}\right)^{2} \sum_{m=1}^{k}\left(Z^{t_{-j}}\left(f_{j_{m}}\right)-\overline{Z^{t_{-j}}\left(f_{j}\right)}\right)^{2}}}
\end{aligned}
$$

where, $Z^{t_{-u}}\left(f_{p_{q}}\right)$ represents the attribute value of the $q^{t h}$ sample point, representing the feature $f_{p}$ at time instance $t_{-u}$, and $\overline{Z^{t}-u\left(f_{p}\right)}$ is the average attribute value of the feature $f_{p}$ over $k$ sample points at the time instance $t_{-u}$.

\section{REV-SEMK $K_{\mathrm{TS}}$ : REVERSE TIMES-SERIES SEMANTIC KRIGING}

This section presents the detailed description of the proposed reverse times-series semantic kriging $\left(\operatorname{Rev}-\mathrm{Sem} \mathrm{K}_{\mathrm{ts}}\right)$ framework. The overall process flow of the framework is depicted in Figure 3. In this framework, the meteorological data and the $L U L C$ distribution of the terrain are considered as the input. After quantification of the semantic knowledge (Bhattacharjee et al., 2014), the temporal and spatial inter-relationships among the meteorological attributes and the different $L U L C$ classes are evaluated. Considering the 3D spatio-semantic and temporal-semantic semivariogram models, the future $L U L C$ distribution pattern is forecasted using the proposed approach $\mathrm{Rev}-\mathrm{Sem} \mathrm{K}_{\mathrm{ts}}$. It can be considered as the multivariate extension of $S e m K_{\mathbf{t s}}$ technique (Bhattacharjee and Ghosh, 2015).

This work assumes that if there $N$ sampled locations in past time instances, any $i^{\text {th }}$ sample point can be characterized as $\left(x_{i},\left\{Z_{1_{i}}\right.\right.$, $\left.\left.Z_{2_{i}}, \ldots, Z_{p_{i}}\right\}, L C_{i}, t_{-i}\right)$. That is the sampled location $x_{i}$, measured at the past time instance $t_{-i}$. The $p$ number of meteorological attributes are measured as $Z_{1_{i}}, Z_{2_{i}}, \ldots, Z_{p_{i}}$, at the sample point $x_{i}$, and its corresponding land-cover $L C_{i}$ (or, $f_{i}$ ) is also known. Here, for our analysis, we have considered two meteorological attributes, $L S T$ and $N D V I$, hence $p=2$. For the forecasting of $L C_{0}$ at the interpolation point $x_{0}$, only past land-cover information could have been used. However, incorporation of other meteorological knowledge may yield more accurate estimation. The proposed framework extends the times-series semantic kriging $\left(\right.$ SemK $\left._{\mathbf{t s}}\right)$ (Bhattacharjee and Ghosh, 2015) in multivariate domain, where multiple secondary attributes are considered for predicting the primary $L U L C$ information of the terrain. The general assumption in multivariate scenario is that the secondary attributes must be correlated with the primary and are estimated jointly utilizing a BLUE (best linear unbiased estimator) (Henderson, 1975). In univariate scenario, the estimation equation of semantic kriging can be given as:

$$
\hat{Z}\left(x_{0}\right)=\sum_{i=1}^{N} w_{i} Z\left(x_{i}\right)
$$

where $\hat{Z}\left(x_{0}\right)$ is the estimated primary attribute value at the prediction point $\left(x_{0}\right), N$ is the total number of interpolating points, $x_{i}$ is the attribute values at the $i^{t h}$ interpolating point, and $w_{i}$ is the weight assigned to each of the interpolating point. In multivariate semantic kriging, as two time-series $\left[L S T\left(Z_{1}\right)\right.$ and NDVI $\left.\left(Z_{2}\right)\right]$ have been considered as the secondary attributes for the estimation, the two multivariate estimation equations are given as follows:

$$
\begin{aligned}
& \hat{Z}\left(x_{0}\right)=\sum_{i=1}^{N} w_{1_{i}} Z\left(x_{i}\right) \text { such that: } \hat{Z}_{1}\left(x_{0}\right)=\sum_{i=1}^{N} w_{1_{i}} Z_{1_{i}} \\
& \hat{Z}\left(x_{0}\right)=\sum_{i=1}^{N} w_{2_{i}} Z\left(x_{i}\right) \text { such that: } \hat{Z}_{2}\left(x_{0}\right)=\sum_{i=1}^{N} w_{2_{i}} Z_{2_{i}}
\end{aligned}
$$

Being an extension of ordinary kriging, both the above equations are constrained by following conditions: $\sum_{i=1}^{N} w_{1_{i}}=1$ and $\sum_{i=1}^{N} w_{2_{i}}=1$ (as unbiased estimator).

The $w_{p_{i}}$ is the weight assigned to the interpolating points, calculated from the semivariogram model. In spatial context, a plot of semivariance versus distance between known sample points is termed as semivariogram. In $\operatorname{Rev}$-Sem $K_{\mathrm{ts}}$ framework, the relationship between meteorological attributes and the $L U L C$ classes is determined using these semivariogram models. Therefore, the concept of semivariogram is further extended in semantic and temporal dimension, resulting spatio-semantic and temporal-semantic semivariogram models. In our application, the prediction attribute $Z$ being the land-use/ land-cover of the terrain, the quantification of this knowledge is carried out using the methods as specified in Section 3. Once this knowledge is quantified, the corresponding changes in primary attribute with respect to the secondary in space and time domain is evaluated using semivariogram models. Hence, for the two individual auxiliary time-series, the spatiosemantic and temporal-semantic semivariogram models are depicted in Figures 4, 5, 6, 7, respectively. These semivariograms are created by taking the distance/temporal lag ( $\mathrm{X}$ axis) and the change of meteorological attribute ( $\mathrm{Y}$ axis) as the independent variables, and the change of $L U L C$ as the dependent variable ( $\mathrm{Z}$ axis).

However, the knowledge of both the meteorological attributes can also be used in order to evaluate how the change of $L U L C$ takes place with respect to the change in both the attributes together. In that case, the semivariograms between the two or more semivariograms (cross-semivariogram) (Vauclin et al., 1983) should be modeled. Figures 8 and 9 depict the change in $L U L C$ with respect to both $L S T$ and NDVI together, in spatial and temporal domain, respectively.

Once these semivariograms are modeled, the $S e m K_{t s}$ approach is 


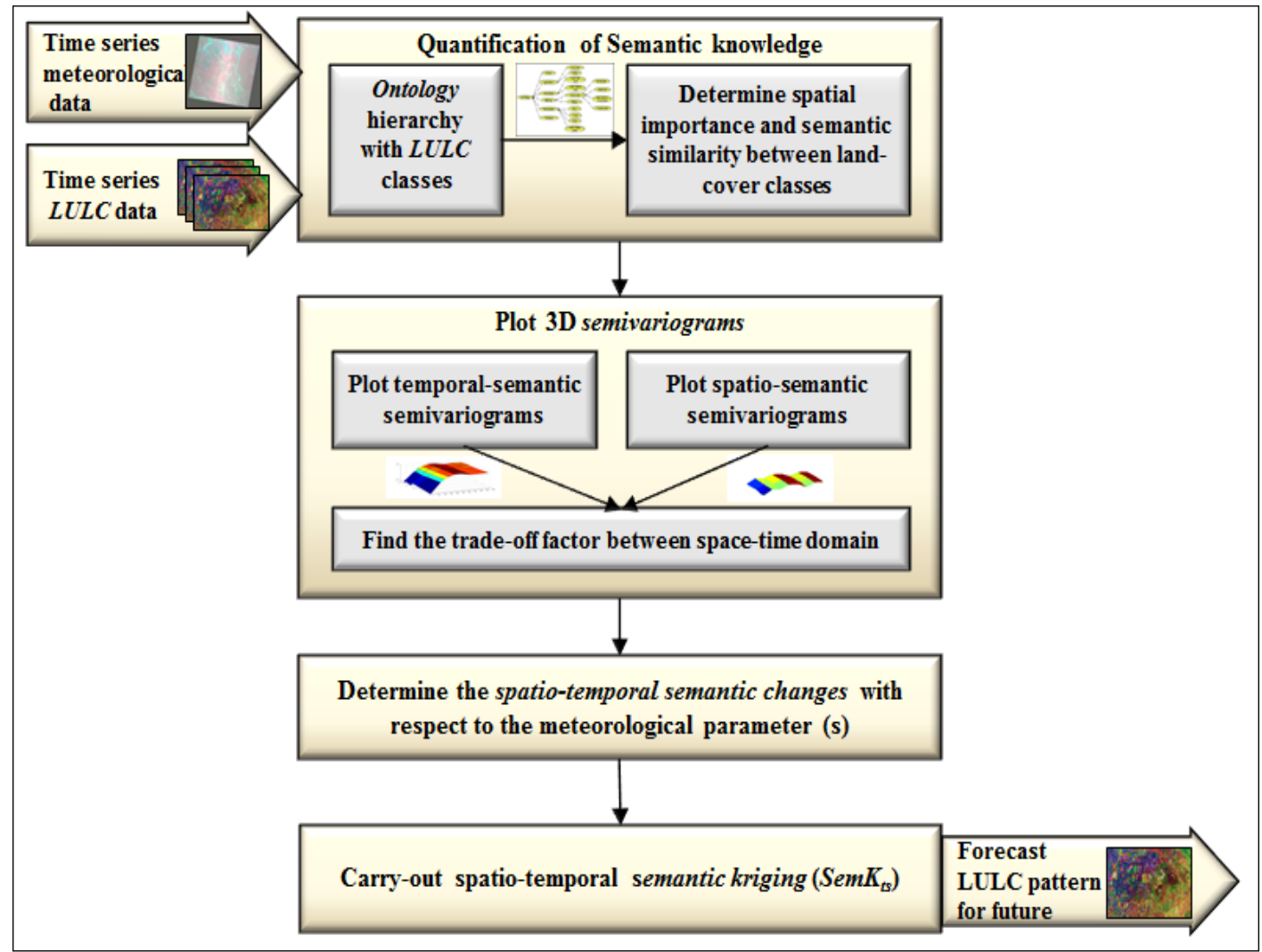

Figure 3. Rev-Sem $K_{\text {ts }}$ Framework

carried out. The traditional spatio-temporal semivariance matrices, $\mathbf{C}^{\mathbf{t}}$, also referred as $\mathbf{W}_{1}^{\mathbf{t}}$ (with respect to the sampled locations), and $\mathbf{D}^{\mathbf{t}}$ (with respect to the sampled and unsampled locations) are modified with the semantic semivariogram measures, resulting $\mathbf{C}^{\text {ts }}$ and $\mathbf{D}^{\text {ts }}$ matrices for $L U L C$, respectively. Each element of the two matrices, i.e., $\mathbf{C}^{\mathbf{t}}{ }_{i j}$ in $\mathbf{C}^{\mathbf{t}}$ and $\mathbf{D}^{\mathbf{t}}{ }_{0 i}$ in $\mathbf{D}^{\mathbf{t}}$ are modified as: $C_{i j}^{t s}=\Delta f_{i j}$ and $D_{0 i}^{t s}=\Delta f_{0 i}$, where $\Delta f_{p q}$ denotes the amount of change in $L U L C$ as $\left(S I_{p q}^{t s} * S S_{p q}\right)$. Hence, as already derived in (Bhattacharjee et al., 2014), the weight vector produced by proposed $\operatorname{Rev}-\operatorname{Sem} K_{\mathrm{ts}}$ framework is given as: $\mathbf{W}_{\mathbf{R e v}-S_{\text {SemK }}}=\mathbf{C}^{\mathbf{t s}-1}\left[\mathbf{D}^{\mathbf{t s}}-\lambda^{t s} \mathbf{1}\right]$, where, $\lambda^{t s}$ is the timeseries Lagrange multiplier of Rev-SemK $K_{t s}$.

\section{EXPERIMENTAL RESULTS}

For the performance analysis of the proposed approach, an empirical experimentation has been carried out with real meteorological data, for the study region Kolkata (a metropolitan city in India, with central coordinate: $\left.22.567^{\circ} \mathrm{N} 88.367^{\circ} \mathrm{E}\right)$. The satellite remote sensing imagery (Landsat ETM+,) offered by United States Geological Survey (USGS) ${ }^{1}$, have been used for this study. These imagery are processed further with the standard satellite image processing tools to obtain the land surface temperature $(L S T)$, normalized difference vegetation index (NDVI) data of the terrain. A supervised classification on the satellite imagery yields the $L U L C$ distribution map of the study region. The signature of six different land-cover classes have been considered for classifying the terrain, such as: forest, agriculture, wastelands, built-up, waterbodies, and wetlands. For the time-series analysis, eleven years data have been considered, for the duration 2000-2010. In order to best fit the semivariogram models, past ten years data have been used (duration 2000-2009) for forecasting the $L U L C$ distribution map in the year 2010 .

\footnotetext{
${ }^{1}$ http://www.usgs.gov/
}

The forecasting have been carried out for some unsampled locations of the terrain by the proposed approach $\operatorname{Rev}-\operatorname{Sem} K_{t s}$. Two zones in the region Kolkata are further subdivided into five subzones, where the predictions have been carried out separately. Three types of external drift have been considered, such as: $L S T$, $N D V I$, and both $L S T$ and NDVI. One standard error metric is considered to quantify the accuracy in prediction, namely root mean square error (RMSE). It may be observed from the case study that the proposed approach with the external drift of more than one correlated meteorological attributes forecast the future $L U L C$ distribution pattern better than other two cases (considering the drift with single attribute).

As mentioned, two spatial zones have been considered from the whole study area, as shown in Table 1 (the bounding box is specified as: [lower left corner, upper right corner]). The forecasted $L U L C$ imagery of both the zones are also depicted in Table 1. The actual $L U L C$ image is shown, along with the predicted imagery by Rev-SemK $K_{t s}$, using the drift of $L S T, N D V I$, and both $L S T$ and $N D V I$, respectively. It has been found that the Rev-SemK $K_{t s}$, with more number of meteorological attribute information, generates better forecasted image and results in higher PSNR (peak signal-to-noise ratio), compared to single attribute drift $(\approx 10-15$ dB higher).

\section{CONCLUSION}

Urban and city planning have drawn concern in the various fields of study for the last few decades. It primarily focuses on the distribution pattern of land-use/ land-cover $(L U L C)$ of the terrain. Hence, anticipating the future behavioral change of $L U L C$ is one of the major research challenges in the field of remote sensing and GIS. This work focuses on forecasting the future $L U L C$ pattern of a region, based on different correlated attribute information. The 


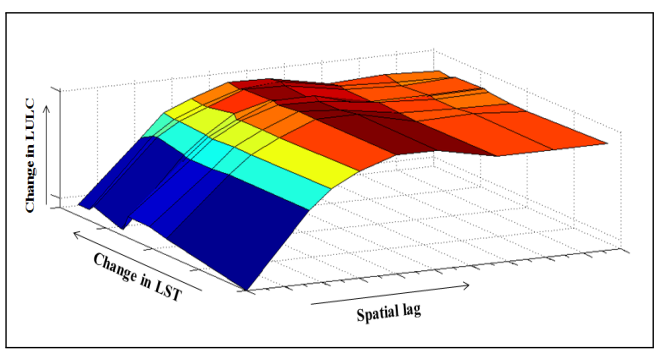

Figure 4. Spatio-semantic semivariogram with respect to $L S T$

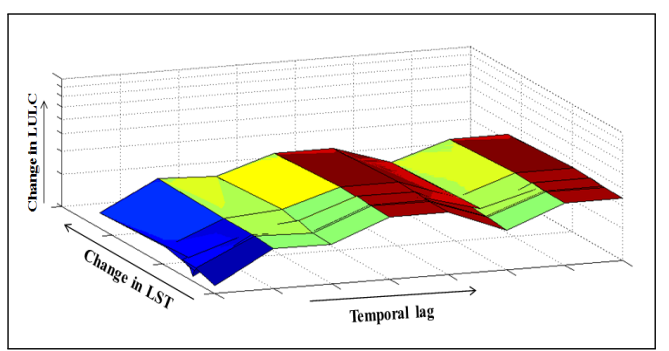

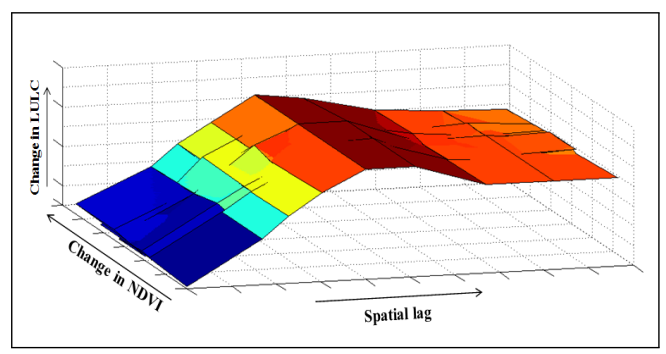

Figure 5. Spatio-semantic semivariogram with respect to NDVI

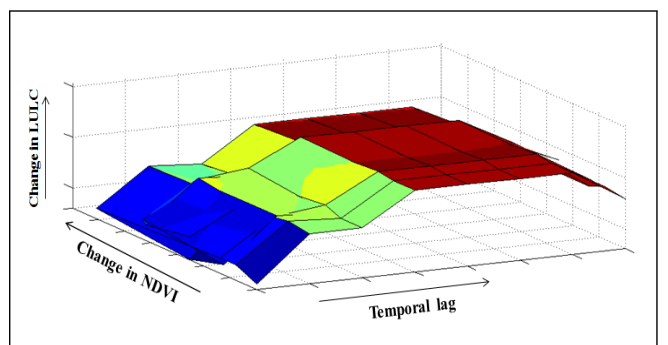

Figure 6. Temporal-semantic semivariogram with respect to LST Figure 7. Temporal-semantic semivariogram with respect to NDVI

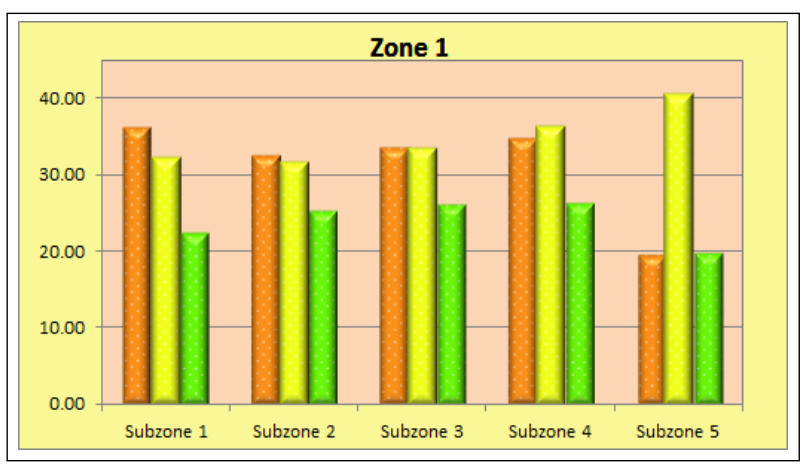

(a) RMSE for zone 1

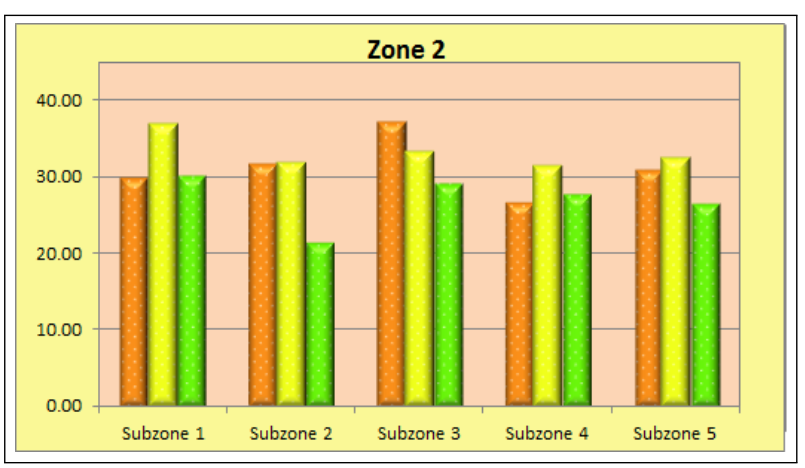

(b) RMSE for zone 2

Figure 10. Prediction error reported using RMSE for five exclusive subzones in two zones

proposed framework, $R e v-S e m K_{t s}$, learns the past behavior of the selected meteorological attributes, for example, land surface temperature, normalized difference vegetation index, etc. Based on the inter-relationships of the past $L U L C$ pattern with these correlated attributes, its future trend is predicted. The proposed Rev$S e m K_{t s}$ is based on the notion of semantic kriging, and can be considered as the multivariate extension of its one of the variants, $S e m K_{t s}$. The case study with real meteorological data shows the efficacy of the proposed method and proves that the incorporation of more correlated attribute information of the terrain enhance the prediction accuracy of $L U L C$ pattern. This framework can be extended further for statistically choosing the appropriate meteorological attributes which are positively correlated with $L U L C$, and for forecasting other semantic knowledge of the terrain as well.

\section{ACKNOWLEDGMENT}

This work is partially supported by the Department of Science and Technology, New Delhi, India, under INSPIRE program.

\section{REFERENCES}

Bhattacharjee, S. and Ghosh, S. K., 2014. Performance evaluation of semantic kriging: A Euclidean vector analysis approach. IEEE Geoscience and Remote Sensing Letters 12(6), pp. 11851189.
Bhattacharjee, S. and Ghosh, S. K., 2015. Time-series augmentation of semantic kriging for the prediction of meteorological parameters. In: 28th IEEE International Geoscience and Remote Sensing Symposium (IGARSS 2015), IEEE.

Bhattacharjee, S., Dwivedi, A., Prasad, R. R. and Ghosh, S. K., 2012. Ontology based spatial clustering framework for implicit knowledge discovery. In: Annual IEEE India Conference (INDICON), pp. 561-566.

Bhattacharjee, S., Mitra, P. and Ghosh, S. K., 2014. Spatial interpolation to predict missing attributes in GIS using semantic kriging. IEEE Transactions on Geoscience and Remote Sensing 52(8), pp. 4771-4780.

Ellis, E. A. and Porter-Bolland, L., 2008. Is community-based forest management more effective than protected areas?: A comparison of land use/land cover change in two neighboring study areas of the central Yucatan Peninsula, Mexico. Forest Ecology and Management 256(11), pp. 1971-1983.

Henderson, C. R., 1975. Best linear unbiased estimation and prediction under a selection model. Biometrics pp. 423-447.

Hengl, T., Heuvelink, G. B., Tadić, M. P. and Pebesma, E. J., 2012. Spatio-temporal prediction of daily temperatures using time-series of MODIS LST images. Theoretical and applied climatology 107(1-2), pp. 265-277. 


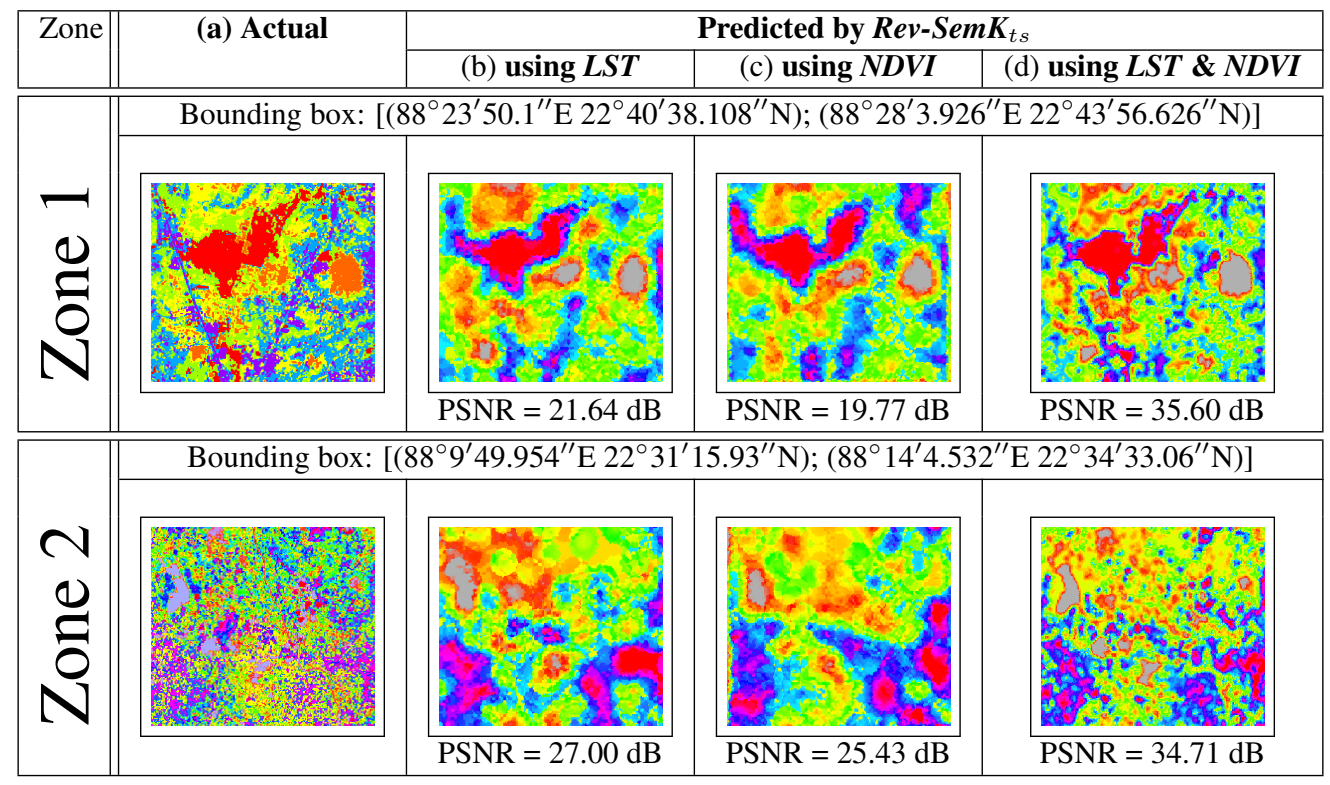

Table 1. Comparison study with (a) actual $L U L C$ distribution imagery, and the predicted imagery (b) using $R e v-S e m K_{t s}$ with $L S T$, (c) $R e v-S e m K_{t s}$ with NDVI, (d) Rev-SemK $K_{t s}$ with $L S T \& N D V I$

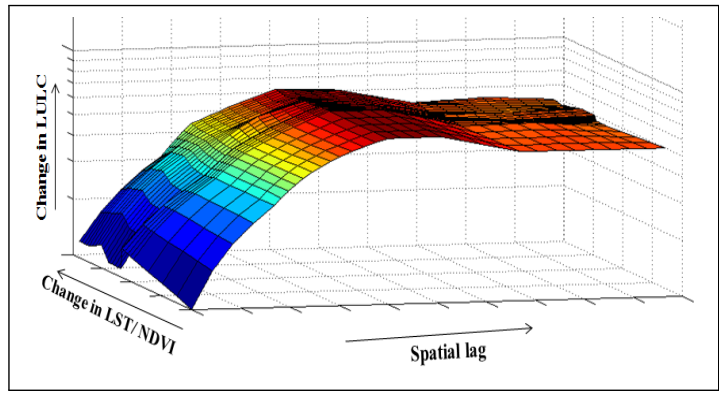

Figure 8. Spatio-semantic cross-semivariogram with respect to LST and NDVI

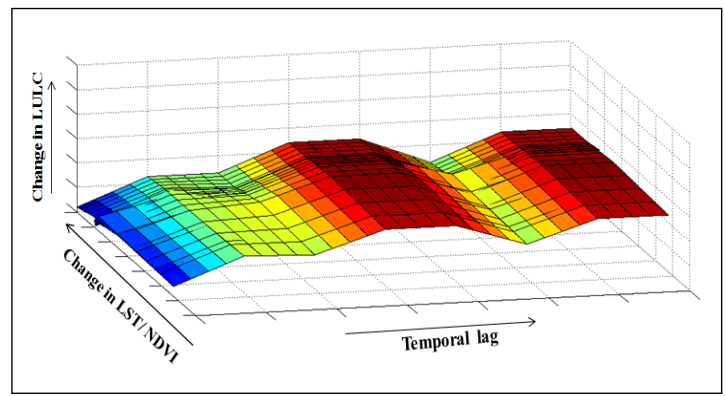

Figure 9. Temporal-semantic cross-semivariogram with respect to $L S T$ and NDVI

Hoek, G., Beelen, R., de Hoogh, K., Vienneau, D., Gulliver, J., Fischer, P. and Briggs, D., 2008. A review of land-use regression models to assess spatial variation of outdoor air pollution. Atmospheric Environment 42(33), pp. 7561-7578.

Humme, A., Lindenbergh, R. and Sueur, C., 2006. Revealing celtic fields from LIDAR data using kriging based filtering. In: Proceedings of the ISPRS Commission V Symposium.

Janssen, S., Dumont, G., Fierens, F. and Mensink, C., 2008. Spatial interpolation of air pollution measurements using corine land cover data. Atmospheric Environment 42(20), pp. 4884-4903.
Lambin, E. F. and Geist, H. J., 2006. Land-use and land-cover change. Local Processes and Global Impacts p. 222.

Mahmood, R., Quintanar, A. I., Conner, G., Leeper, R., Dobler, S., Pielke Sr, R. A., Beltran-Przekurat, A., Hubbard, K. G., Niyogi, D., Bonan, G. et al., 2010. Impacts of land use/land cover change on climate and future research priorities. Bulletin of the American Meteorological Society 91(1), pp. 37-46.

Manning, C. D., Raghavan, P. and Schütze, H., 2008. Introduction to Information Retrieval. Vol. 1, Cambridge University Press.

Mendiratta, N., Kumar, R. S. and Rao, K. S., 2008. Standards for bio-geo database. Technical Report 1, Natural Resources Data Management System (NRDMS) Division, New Delhi, India.

Muñoz-Villers, L. E. and López-Blanco, J., 2008. Land use/cover changes using landsat TM/ETM images in a tropical and biodiverse mountainous area of centraleastern mexico. International Journal of Remote Sensing 29(1), pp. 71-93.

Petrişor, A.-I., Ianoş, I. and Tălângă, C., 2010. Land cover and use changes focused on the urbanization processes in romania. Environmental Engineering and Management Journal 9(6), pp. $765-771$.

Schilling, K. E., Chan, K.-S., Liu, H. and Zhang, Y.-K., 2010. Quantifying the effect of land use land cover change on increasing discharge in the upper mississippi river. Journal of Hydrology 387(3-4), pp. 343-345.

Sertel, E., Ormeci, C. and Robock, A., 2011. Modelling land cover change impact on the summer climate of the marmara region, turkey. International Journal of Global Warming 3(1), pp. 194-202.

Shukla, J., 1998. Predictability in the midst of chaos: A scientific basis for climate forecasting. science 282(5389), pp. 728-731.

Stein, M. L., 1999. Interpolation of Spatial Data: Some Theory for Kriging. Springer Verlag.

Tobler, W. R., 1970. A computer movie simulating urban growth in the detroit region. Economic geography 46, pp. 234-240. 
ISPRS Annals of the Photogrammetry, Remote Sensing and Spatial Information Sciences, Volume II-4/W2, 2015 International Workshop on Spatiotemporal Computing, 13-15 July 2015, Fairfax, Virginia, USA

Vauclin, M., Vieira, S., Vachaud, G. and Nielsen, D., 1983. The use of cokriging with limited field soil observations. Soil Science Society of America Journal 47(2), pp. 175-184. 